\title{
Adapting Distance Relay Using Artificial Neural Networks
}

\author{
M. F. AL-Kababjie \\ Semaa M. AL-Taee \\ Electrical Engineering Department - College of Engineering- Mosul University
}

\begin{abstract}
A universal distance relay that has merits over conventional relays is designed. This relay has the ability to adapt more than one working characteristic by using an artificial neural networks technique(ANN)of the type Modified Multi-Layer Perceptron (MMLP) which uses additional nodes at the input layer to realize an adaptation through the required modifications on the distance relay characteristics. The inputs of the added nodes referred to the factors that affect the distance relay characteristic such as: fault resistance, power transfer, mutual inductance and locations of feeding sources in the system. A Matlab computer programming was used to simulate different power systems of $(400,132) \mathrm{KV}$ to obtain impedance characteristic data, needed to train and test the ANN. A laboratory power system ( $220 \mathrm{~V})$ has been operated as a computer simulation model and practically under the same conditions. The designed universal relay gives good performance when tested on a computer model of this system . The small difference between the practical results and that of simulation, encourage the use of designed relay to protect transmission lines in reality.
\end{abstract}

Keywords: Artificial neural network, Adaptive Distance Relay characteristics
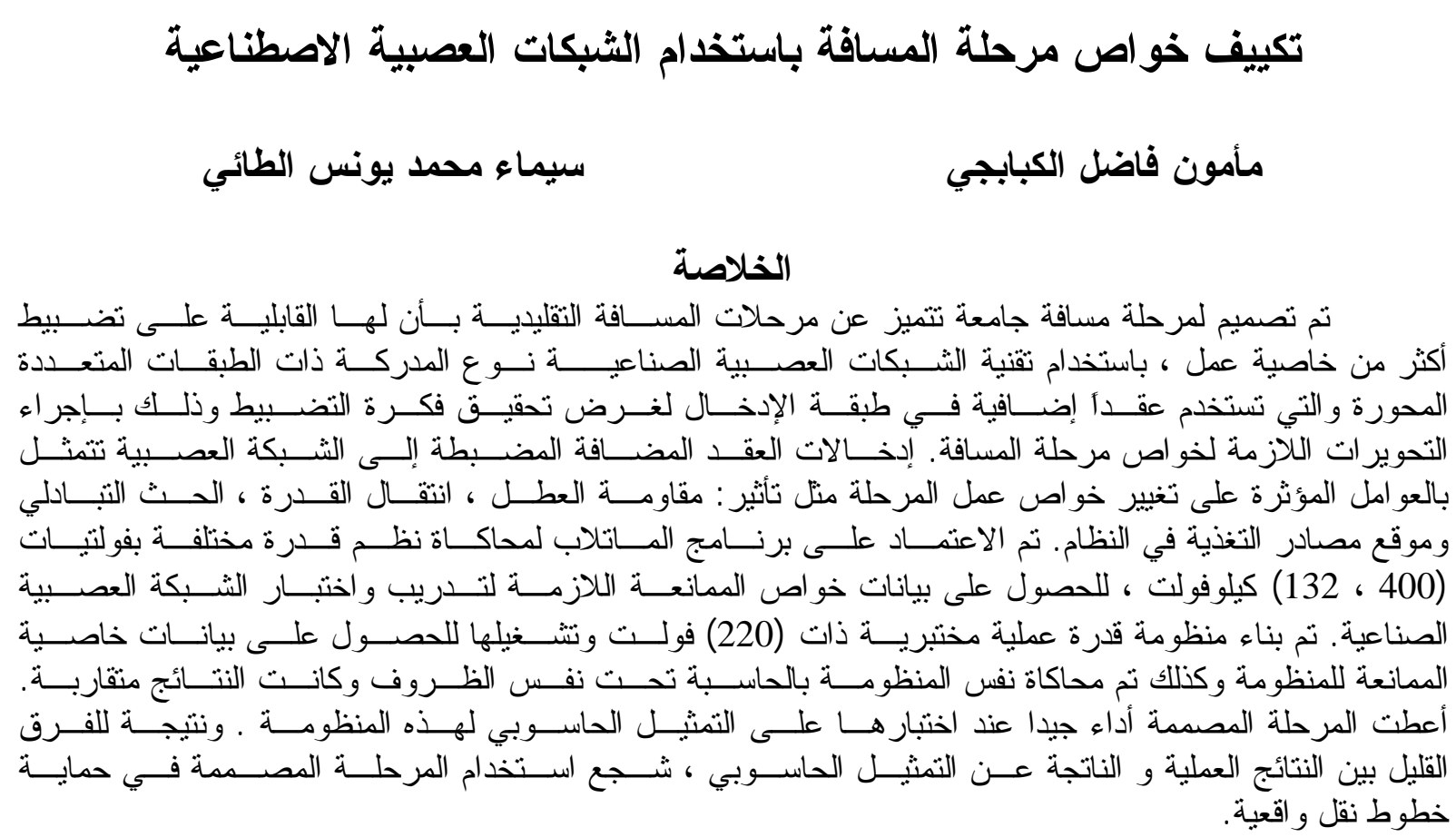


\section{Al-Rafidain Engineering \\ Vol.17 No.3 \\ June 2009}

\section{Introduction:}

An overhead transmission line is one of the main components in every electric power system. The transmission line is exposed to the environment and the possibility of experiencing fault on the transmission line is generally higher than that on the other main component [1]. Distance protection relays are commonly used for protection of transmission line .Since the impedance of transmission line is proportional to its length; such a relay is based on impedance calculation. Although the fundamentals of distance protection were enunciated many years ago, theoretical principles as well as practical application of distance protection are still common topics of investigation [2]. Line to ground fault is the most common fault. When a fault occurs on an electrical transmission line it is very important to detect it and to find its location in order to make necessary repairs and to restore power as soon as possible. The time needed to determine the fault point along the line will affect the quality of the power delivery [3]. There is some factors which need to be considered while choosing relay characteristics can be listed as:

1-There are adverse effects of uncontrollable variables such as resistance in fault path with possible reactive component, variable fault location.

2- Source X/R ratio.

3- Pre-fault load flow also affects the impedance seen by the relay.

4- Mutual inductance.

In order to deal with the above-mentioned problems the concept of adaptive relaying has been studied. Digital distances relay implementing adaptive features susceptible to the abovementioned adverse effects, which in turn reduce the mal-operation of the relay [4][5][6][7]. This work explores further how adaptive relaying can be achieved by a modified multilayer perception model (MMLP). This work merits on the research (6) by adding two nodes at the inputs of relay to process many effects. So the design relay become more generally to protect many transmission lines, therefore the designed relay protect most of transmission lines of the Iraqi National Super Guide (INSG) $(400 \mathrm{Kv})$ and some transmission line of $(132 \mathrm{Kv})$, depending on the additional nodes, .The modification of the characteristic in accordance to system conditions can be obtained by introducing additional nodes at the input layer which is flagged 'on' depending on the systems status. This adaptation provides facilities to protect more than one transmission line in difference power systems. line model that includes the fault resistance and remote-end infeed.

\section{Adaptive Relaying:}

Adaptive protection, as 'Adaptive protection is a protection philosophy which permits and seeks to make adjustment to various protection functions in order to make them more attuned to prevailing power system conditions' [6][8]. The concepts of adaptation in general cover almost all protection schemes. Dependability is correct operation of relay whereas security involves no incorrect

operations. For EHV lines dependability is more important whereas for distribution network security is of prime importance.

\section{Artificial Neural Network In Adaptive Relay:}

Artificial Intelligent (AI) is a subfield of computer science that investigates how the through and action of human beings can be mimicked by machines. The AI includes not only the ability to make rational decisions, but also to deal with missing data, adaptive to existing situations and improve itself in the long time horizon based on the accumulated experience. One of the major families of AI techniques is Artificial Neural Network (ANN) [9]. 
Artificial neural network based technology, which is inspired by biological neural networks, has developed rapidly in the previous decade and has been applied in power system protection applications. Specific applications include direction discrimination for protecting transmission lines [10][11]. A very interesting alternative to the more conventional solutions for adaptive relay characteristics is the use of ANN. Distance relaying of high resistance earth faults with remote infeed give rise to measuring problems mainly because of their non-liner nature [7]. ANN seems to be a very attractive tool for this problem because it contain suitable adapting feature in the field of power system protection. Neural network can be fully applied for pattern recognition and classification, it has been widely investigated for transient classification (including faults) [12][13].

\section{Systems Model for Distance Relay Studies:}

External conditions of protected line influence relay performance. To demonstrate this effect several $400 \mathrm{kv}$ and $132 \mathrm{kv}$ systems models has been considered .This $400 \mathrm{kv}$ model is The Iraqi National Super Guide (INSG) system. The system consist of 19 bus bars and 27 transmission lines which are different in its length. The total length of the lines is $3410.2 \mathrm{~km}$. Six generating stations are connected to the guide. Some transmission lines of INSG system are used to obtain the input /output data to train the ANN, and the other used to test it. Fig.(1) shows the single line diagram of the INSG (400kv) system. The diagram shows all the bus bars, the given loads which connected on each bus bar represent the actual values of the bus bars load. While the $132 \mathrm{kv}$ system is a section from northern Iraqi network between Moslnorth bus bar and Rash bus bar as shown in fig.(2). This system consist a generator at two sides, parallel transmission line and load at each side. Because the small distance between the parallel lines in the system the effect of mutual inductance appears on the system of $132 \mathrm{Kv}$.

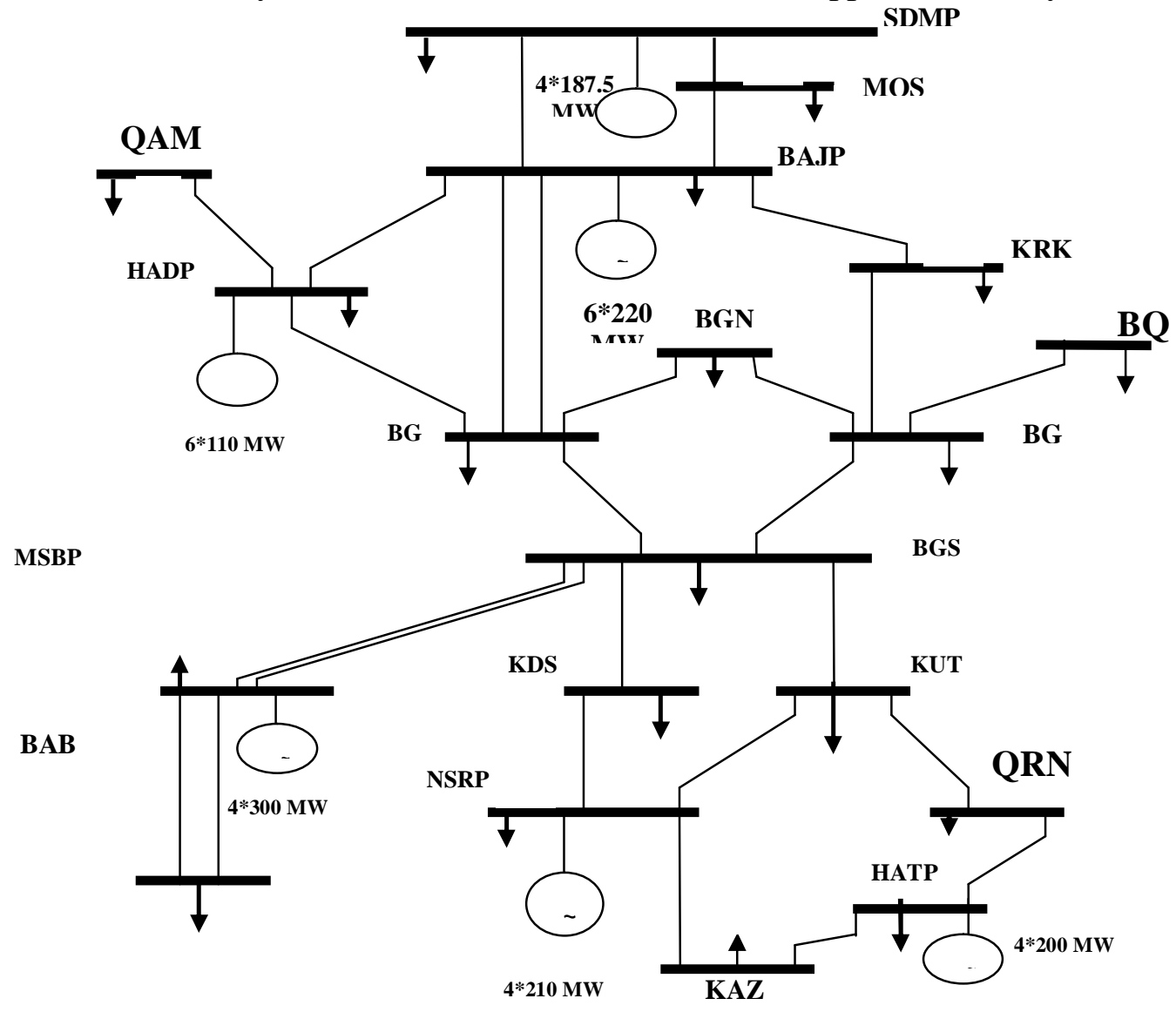

Figure (1): single line diagram of the INSG (400kv) system 


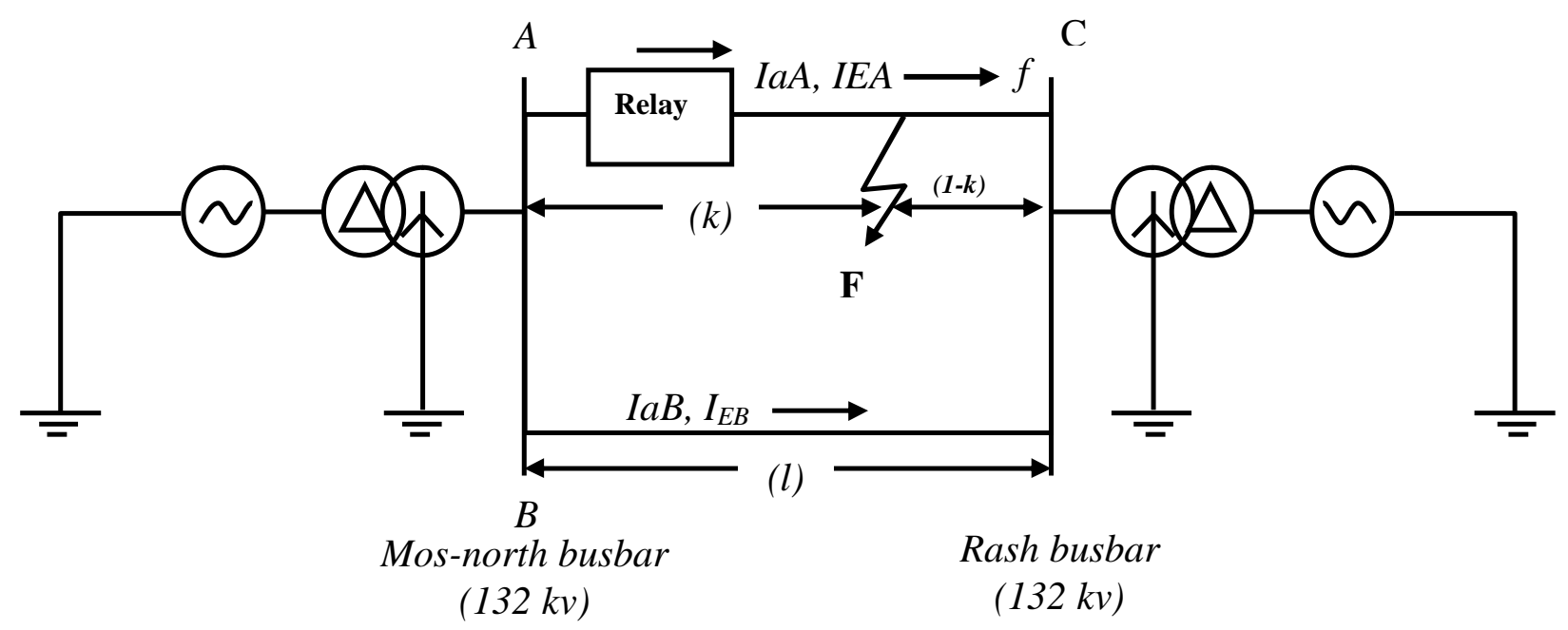

Figure (2): a section from northern Iraqi network (132kv)

\section{Ideal Operating Region of Distance Relay:}

There are many factors affecting the ideal operating region of a distance relay such as fault resistance which affecting on the width operating area of the impedance characteristic, and the load transfer which affecting on vector of the upper line of the characteristic as shown in fig (3). A (MATLAB-Simulink-P.S.B) computer programming used to simulate the considered power systems to obtain impedance characteristics data. These values are the line resistance $(\mathrm{R})$, line reactance $(\mathrm{X})$ and angel of the measured impedance at relay point by applying a single line to ground fault in the first protected zone and with fault resistance values between ' 0 to 20 ' $\Omega$. Under different system conditions and affecting factors, fig (3) shows the resultant relay characteristics needed to train and test the artificial neural network.

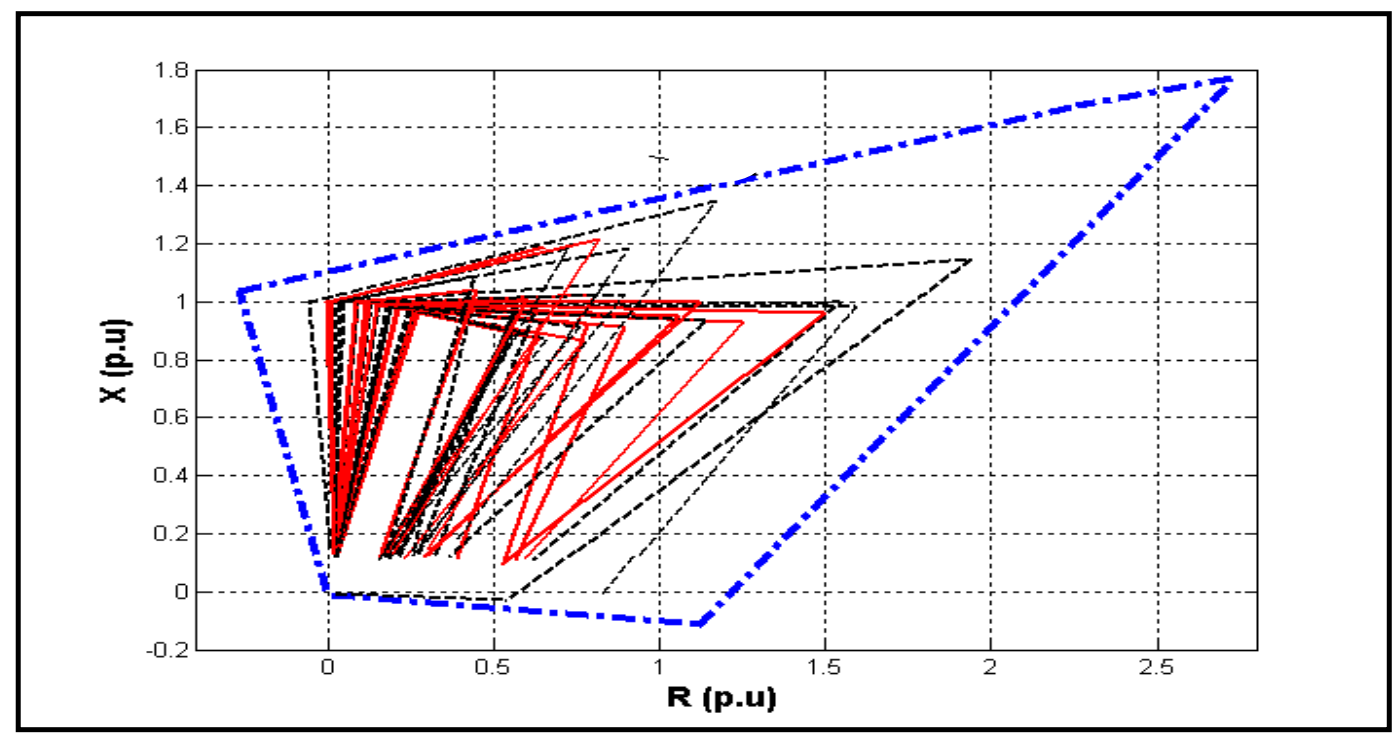

Figure (3) Resultant impedance characteristics for many transmission lines

\section{Adaptation of distance relay characteristic:}

\subsection{Fault resistance and power transfer :}

Distance relay of high resistance earth faults with remote infeed give rise to measuring problems mainly because of their non-linear nature. It has been shown that in conventional distance relay, the impedance measurement at the local end only can be expressed as: 
$\mathbf{Z}_{\mathbf{m}}=\mathbf{k} \mathbf{Z}_{\mathbf{L}}+\Delta \mathbf{Z}$

The measured impedance $\mathbf{Z}_{\mathbf{m}}$ may be larger or smaller than the actual value $\mathbf{Z}_{\mathbf{L}}$ depending on the value of the phasor $\Delta \mathrm{Z}$ for different power system conditions. Consider a two end fed transmission line as shown in fig. (4). A Fault occur at a point on the line is ' $\mathrm{k}$ ' per unit distance from relay location ' $A$ '. Let $R_{F}$ be the fault resistance and $Z_{L}$ be the total line impedance. $I_{1} \& I_{2}$ are the steady state post fault currents flowing into fault from ends A \& B respectively, then the apparent impedance seen by relay ' $A$ ' is:

$Z_{R}=k Z_{L}+\left(1+I_{2} / I_{1}\right) R_{F}$

An error in the impedance measured by the relay ' $A$ ' is equal to $\left(1+I_{2} / I_{1)} R_{F}\right.$.And if the value of this term is large then it might cause the value of the apparent impedance $Z_{R}$ to lie outside the operating zone. The error term when split into the three components can be considered to be:

(i) A rightward shift in $\mathrm{R}-\mathrm{X}$ plant due to the term ' $\mathrm{R}_{\mathrm{F}}$ ' term.

(ii) The second term ' $\mathrm{I}_{2} \cdot \mathrm{R}_{\mathrm{F}} / \mathrm{I}_{1}$ ' which gives the fault a reactive component due to the load angle between the two currents $I_{1} \& I_{2}$ which is positive or negative. This angle depends on the power transfer from the two side of the transmission line.

(iii) The third effect is the power transfer from the two sides of the transmission line that affect on the wide area of the relay characteristic and depend on the magnitude of the infeed current $\mathrm{I}_{1} \& \mathrm{I}_{2}$. This effect also depends on the length in 'km' of the transmission line. [7]

Hence depending upon the problems mention above the relay will either over-reach or under-reach. Fig. (3) show the difference in relay characteristics because the differ of the transmission line location in the (INSG) power system and the affecting factors on the transmission line, namely, fault resistance, power transfer, and the magnitude of the current infeed from the two sides in fault path and the angle between them. Due to the affecting factor each transmission line has a relay characteristic differ on the other by the line angle ' $\theta_{\mathrm{L}}$ ' and the reach point resistance $\left(\mathrm{R}_{\mathrm{d}}\right)$ at $80 \%$ of the length line and $20 \mathrm{ohm}$ fault resistance as shown in fig. (5). Therefore, we shall attempt to compensate this difference in various relay characteristics by resorting to an adaptive relaying scheme. This scheme should modify the relay characteristic to attenuate the affecting factor on the protected transmission line [4].

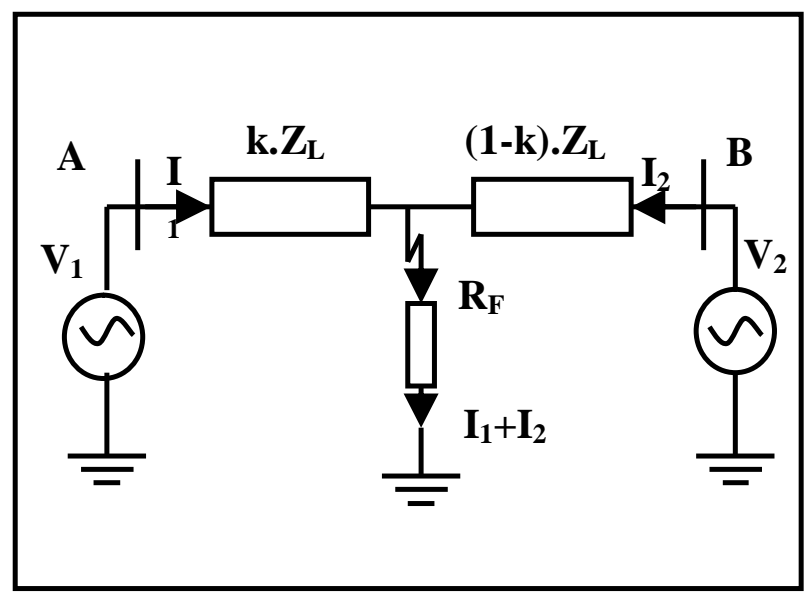

Figure (4) measuring of line impedance with $\mathbf{R}_{F}$

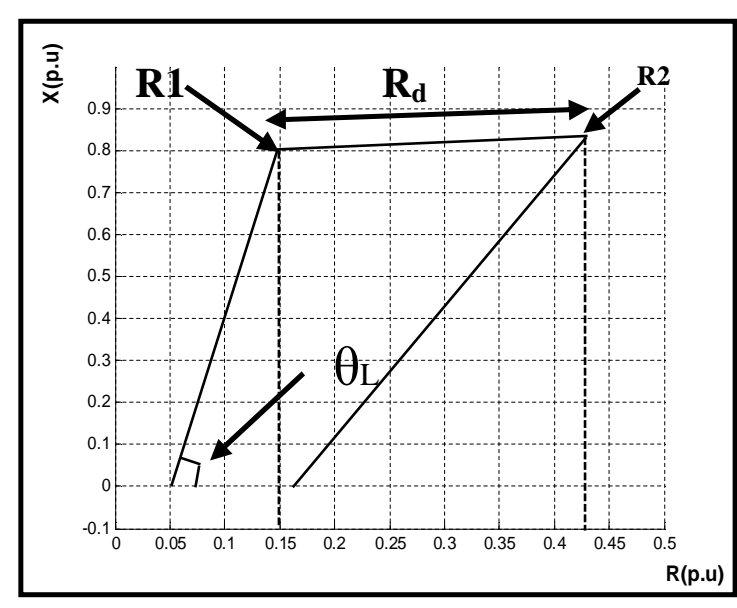

Figure (5) the two additional input on impedance characteristic 


\subsection{Mutual inductance in parallel lines [4][15]}

When overhead lines follow parallel paths, a mutual inductive coupling of the current paths exists. In the case of transposed lines, this effect in the positive and negative sequence may be neglected for all practical purposes (mutual reactance less than 5\% of the selfimpedance). The coupling impedance $\left(\mathrm{Z}_{\mathrm{OM}}\right)$ between the zero sequence systems of the two lines without earth wire is given by the following equation:

$$
\mathrm{Z}_{\mathrm{OM}}=0.15+\left(\mathrm{j} 0.1889 \ell_{\mathrm{n}} \frac{931}{\mathrm{~A}_{\mathrm{L}}}\right)[\Omega / \mathrm{km}] \ldots \ldots . . .(3)
$$

From this it is apparent that the mutual inductance has a logarithmic relationship, and therefore decreases relatively slowly with an increase of the Geometrical Mean Distance $\left(\mathbf{A}_{\mathbf{L}}\right)$ between the two parallel lines. Even with fairly large distance between the linens, mutual coupling is still present. To study this effect on the distance protection measurement that used to protect parallel lines $(132 \mathrm{kv})$, and how to make the necessary adaptation for the distance relay characteristics that affecting and changing with the mutual impedance, we considered a section from northern Iraqi network (132kv) as shown in fig.(2). The considered transmission line in feed from two sides, if lines to ground fault, occur at point ' $F$ ' on the path ' $A$ ' at a distance location ' $\mathrm{K}$ ' from the relay point. Let ' $\mathrm{p}_{\mathrm{ph}}$ ' the phase fault current seen by relay, so the measured impedance $\left(\mathrm{Z}_{\mathrm{R}}\right)$ may now be calculated by using the following equation:

$$
\mathrm{Z}_{\mathrm{R}}=\mathrm{K} \times \mathrm{Z}_{\mathrm{L}}+\mathrm{K} \times\left(\frac{\left(\mathrm{Z}_{\mathrm{OM}} \times \mathrm{I}_{\mathrm{OB}}\right)}{\mathrm{I}_{\mathrm{ph}}+\left(\frac{\mathrm{Z}_{\mathrm{OL}}-\mathrm{Z}_{1 \mathrm{~L}}}{\mathrm{Z}_{1 \mathrm{~L}}}\right) \times \mathrm{I}_{\mathrm{OA}}}\right)[\Omega] \ldots \ldots \ldots \text { (4) }
$$

$\mathrm{Z}_{\mathrm{L}}:$ Is the line Impedance.

$\mathrm{Z}_{\mathrm{OL}}$ :Is the zero sequence line impedance.

$\mathrm{Z}_{1 \mathrm{~L}}$ :Is the positive sequence line impedance.

$\mathrm{I}_{\mathrm{OB}}$ :zero sequence current of line $\mathrm{B}$.

$\mathrm{I}_{\mathrm{OA}}$ :zero sequence current of line $\mathrm{A}$.

It can be seen that the measuring error is dependant on the Geometrical Mean Distance $\left(\mathbf{A}_{\mathbf{L}}\right)$ and on the polarity and magnitude ratio of the current in two path. Fig (6) shows the impedance characteristics that resultant by applying a fault on the line 'AC' with various values of $\left(\mathbf{A}_{\mathbf{L}}\right)(13.4,20,40,60,80)$ meters in the first protected zone. Fig (6 - a) shows the relay working characteristic without taking the effect of the mutual coupling, but the fig (6 - ' $\mathrm{b}, \mathrm{c}, \mathrm{d}$, e and $\mathrm{f}$ ') represent the working characteristics with the effect of mutual with distance $\left(\mathbf{A}_{\mathbf{L}}\right)(80,60,40,20,13.4)(\mathbf{m})$ respectively . This effect causes to increase the values of the measured impedance on its real values (with out the effect of the mutual), so the relay will not operate even if the fault occur inside the protected zone (operate in under reach).

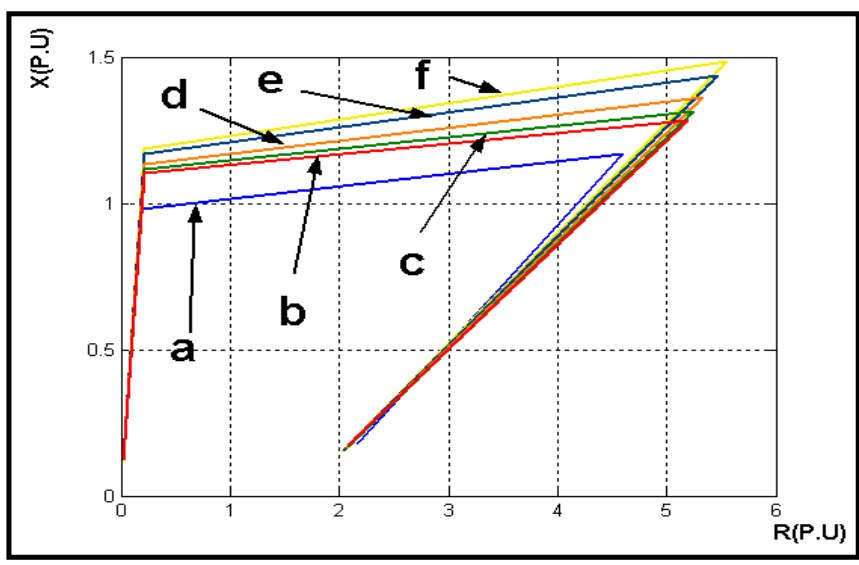

Figure (6) Impedance characteristics 


\section{Modified Multi-layered perceptron (MLP) model for Adaptive Relaying:}

As pointed, we consider two systems, the Iraqi National Super Guide (INSG) power system $(400 \mathrm{kv})$ and a section from northern Iraqi network $(132 \mathrm{kv})$. The goal is to avoid the mal-operation of the relay in protecting transmission line with various working characteristics in different conditions of the power system.

The designed relay consists of Sub-neural networks, 'NN1' for detecting the fault and 'NN2' for detecting its location.

In addition to the regular inputs to a designed ANN relay, additional nodes are used modified MLP (MMLP) model shown in fig (7.a).

The input layer of the Sub-NNs that designed as a relay which protecting the $400 \mathrm{kv}$ system has four inputs: the first two inputs are the line resistance $(R)$ and reactance $(X)$ of the measured impedance. The other inputs considered as required as additional inputs that represent the differ in the working characteristic due to power system condition and affecting factor. The additional input is the

angle of the line impedance $\left(\theta_{\mathrm{L}}\right)$ and the reach point resistance ' $\mathrm{R}_{\mathrm{d}}$ ' (at $80 \%$ line length and $20 \mathrm{ohm}$ fault resistance). The addition inputs to the relay that used to

protect the system that has the effect of the mutual inductance, is the distance $\left(\mathbf{A}_{\mathbf{L}}\right)$, to adapt the impedance characteristics shown in fig (6), then the relay protect the transmission line of $(132 \mathrm{kv})$. The output of the NN1 is ' 1 ' when a fault occurs in the first protection zone and ' 0 ' when a fault occurs outside. The output of the NN2 gives the location of the fault as a percent of the line length (from $0.05 \%-80 \%$ ) by step of $10 \%$.

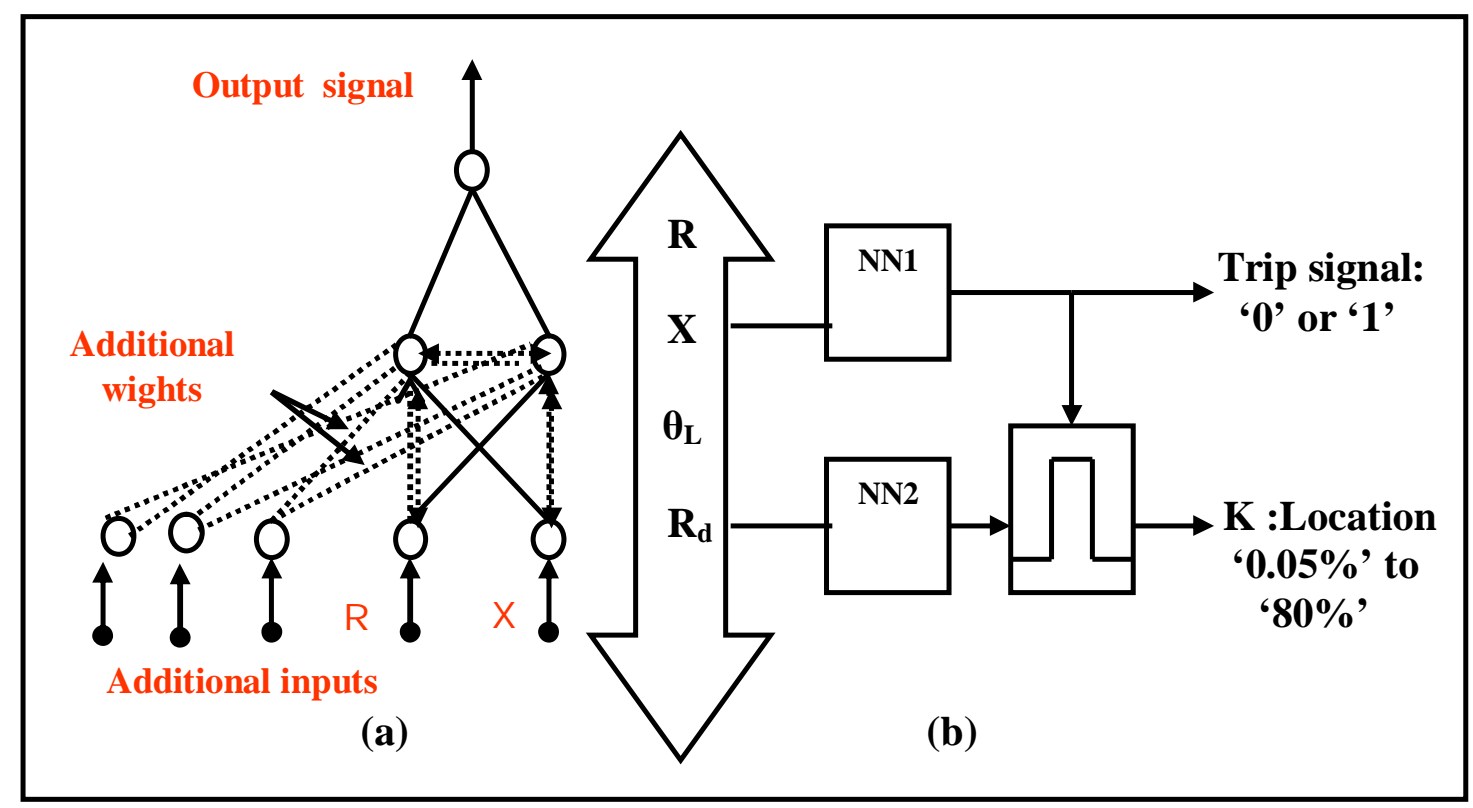

Figure (7) Diagram of the operation meganisim of the ANNs

\section{Training the ANNs:}

The size of the input set is very important for good performance because that the ANN get its information from training. If the data do not cover the full range of operating condition, the system may be performing badly when eployed. Fig (3) show the characteristics form of the data that used to train and test the ANNs. The characteristics data plotted in solid-lines represent the training characteristics.

The back propagation (BP) algorithm is used in this work. It consists of two stages, that is forward and backward propagation [14]. The impedance characteristics shown in fig 


\section{Al-Rafidain Engineering \\ Vol.17 No.3 \\ June 2009}

(6) also entered to the training phase to compensate the effect of mutual inductance on the $132 \mathrm{kv}$ system. The number of the hidden layers and the neuron in each layer affects the accuracy of the non-linear boundary and operating speed. The importance of the MLP is that it is a universal mapper (implements arbitrary input/output maps) when the topology has at least two hidden layers and sufficient number of neurons. This means that rarely we will need to choose topologies with more than two hidden layers ..However, these are existence proofs, so the issue that we must solve as engineers is to choose how many layers and how many neurons in each layer are required to produce good results [13]. Therefore, in this paper we trained the ANNs with more than two hidden layers because of various cases and the complex of input/ output data set. In this work the training phase gives good results when the topologies of ANN has six hidden layers to process a data of 5000 value for each input.

\section{Testing patterns and results:}

Testing transmission lines should be properly selected to represent different fault measurements. Fig. (3) and fig. (6) shows all the characteristics data, the characteristics plotted in dashed-line, which are not entered in training phase, are the results of tested transmission lines while the others were for training. The designed relay has the ability to be used for different transmission system for multi system conditions. Depending upon the additional inputs, the relay adapts and modifies the working characteristic to fit various protected transmission lines. The neural network output can cover many working characteristics with a maximum fault resistance of $20 \Omega$ from the relaying point up to $80 \%$ of line length. The designed relay consider the single line to ground faults only, which represent the majority $(85 \%)$ of the line faults. The relay performance has good enough to detect the fault with small error ratio. During the fault detection, the workings of NN1 \& NN2 are cascaded as shown in fig (7.b).

The error due to fault location is defined as:

\section{Error $(\mathbf{k m})=/ \mathrm{ANN}$ output - Actual fault location $/$}

Where

'ANN output' is the output (in km) of the ANN fault locator, 'Actual fault location' is the real distance the fault in the transmission line (in $\mathrm{km}$ ).

The criterion for evaluating the performance of the fault locaters defined as:

$$
\text { Error }(\%)=[\text { Error }(\mathrm{km}) / \text { length of line }(\mathrm{km})]^{* 100 \%}
$$

The relationships between the fault locations for all the tested transmission lines which differ in length, and the percentage error ratio in fault location for variable values of fault resistances are shown in fig. (8) and fig. (9). Fig. (8) For fault resistance of $7 \mathrm{ohm}$ and (15 50 $7580) \%$ fault locations while fig. (9) Show that relation with fault resistance $15 \mathrm{ohm}$ and at the same locations. It can be seen from these figures the, maximum and minimum percentage errors for fault locators. Error ratio in location is between (0-0.4) \%. 


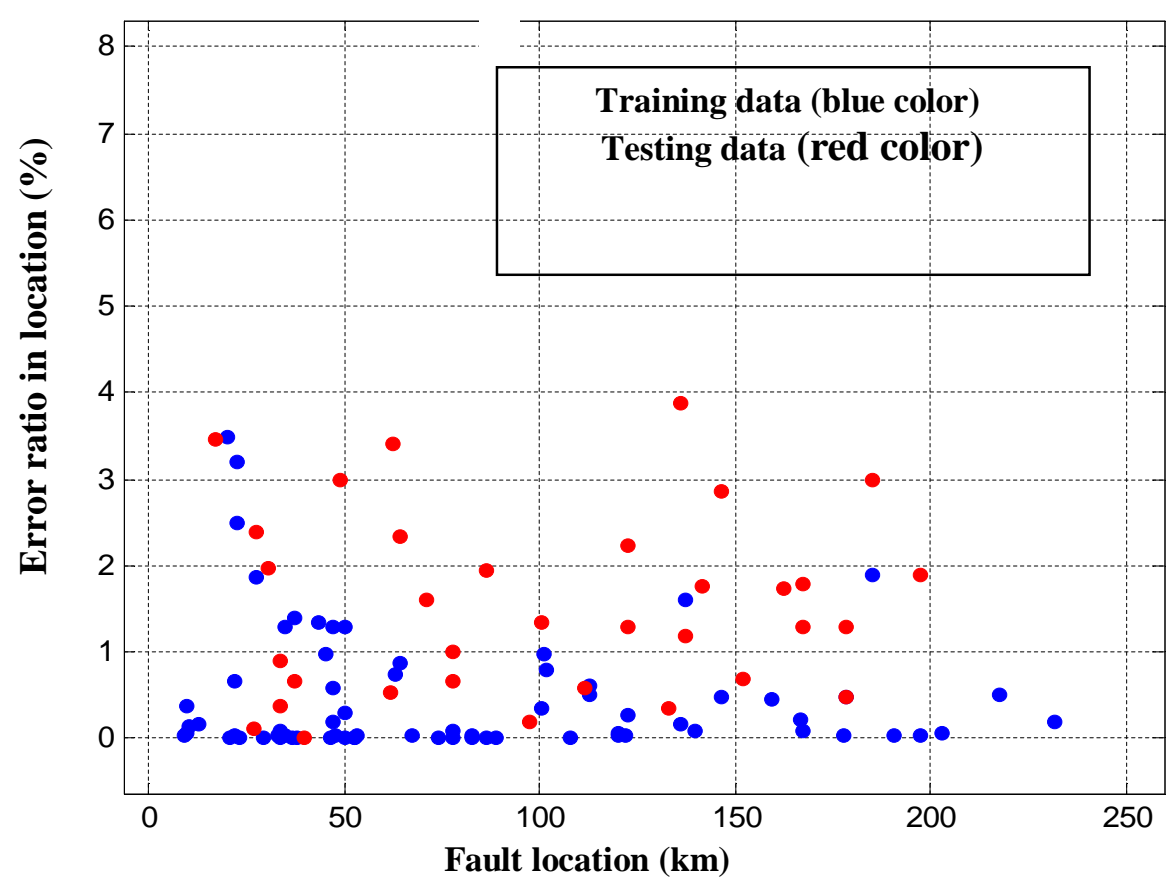

Figure (8)The relation between the percentage error in fault location with the real location in $(\mathrm{km})$ at $R_{F}=7 \mathrm{ohm}$

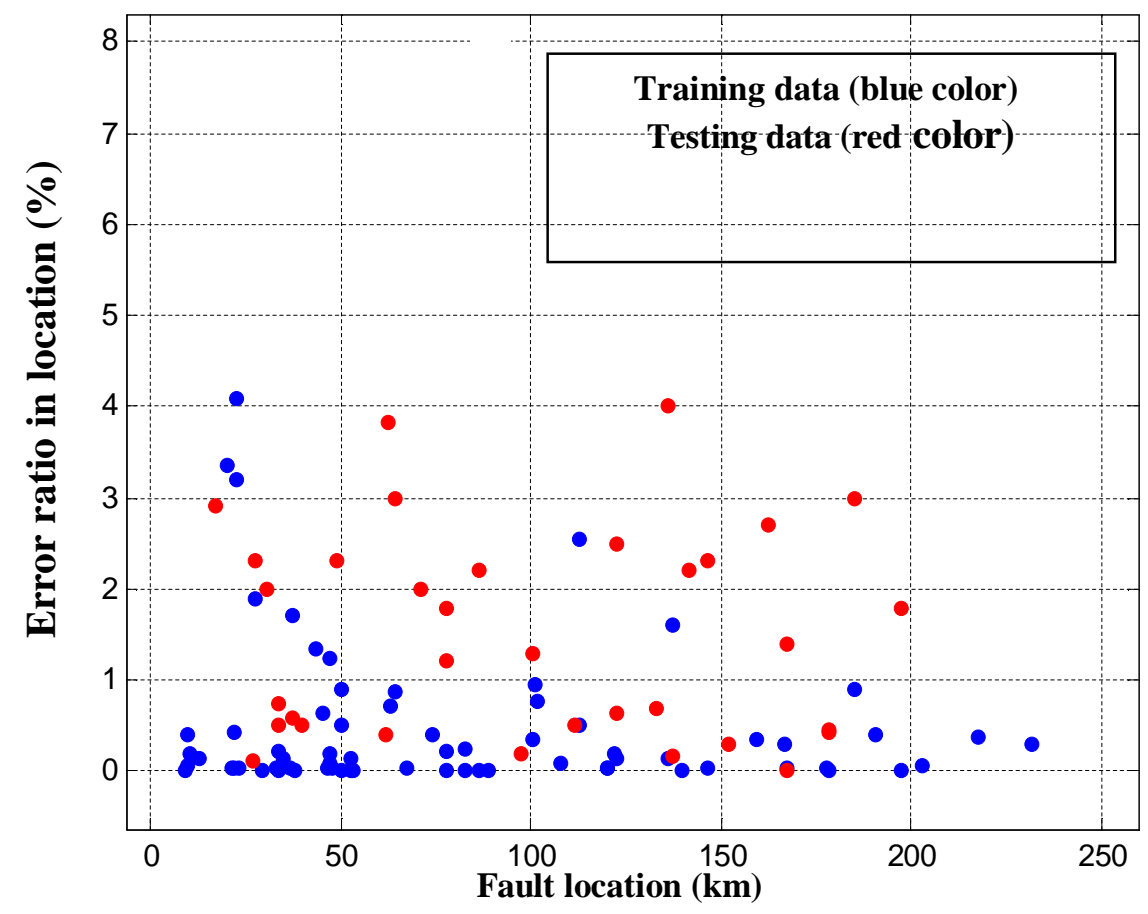

Figure (9) The relation between the percentage error in fault location with the real location in $(\mathrm{km})$ at $R_{\mathrm{F}}=15 \mathrm{ohm}$ 
The designed distance relay gives good results when tested on a laboratory power system in electrical engineering department / Mosul University, as example of real power system .The relay detect the fault at $80 \%$ from the length of the transmission line . Fig (10) show the resultant relay characteristic when applying a fault on the transmission line of the laboratory system when the_system operated practically fig (10 'a \& c') and by simulation model in

MATLAB programming fig (10 'b'). We can see clearly the closeness between the two resultant characteristics

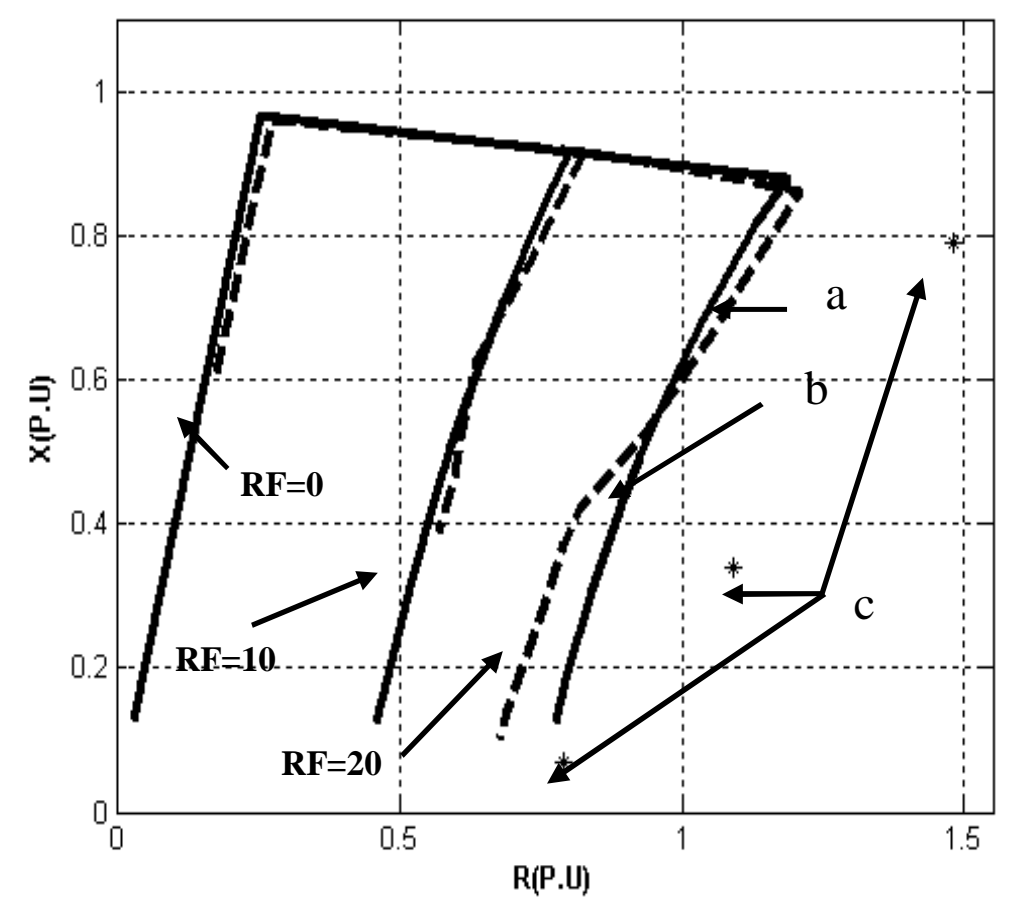

Figure (10) (a \& c) The characteristic from operating laboratory system , (b) the result characteristic simulation model

\section{Conclusion:}

The designed relay testing on most of the transmission power system mentioned. The result reveal that the relay gives a good performance in protection of all transmission lines considered through choosing and adapting the working characteristic that is suitable for the protected transmission line and detect the fault and its location specially for lines data that not entered in training phase. The testing results was with fairly acceptable error ratio, it was found that the higher error ratio to isolate the faulted line in the first zone equal to $3.6 \%$. For detecting the fault location, the higher error ratio in location between the real location and the detected location is not more than 4\%. The Low Pass Filter used in this work estimate the out put signal of the designed relay more accurately. Therefore, unwonted operations of the relay due to miscalculation of impedance could be prevented and this way amore secure relay is obtained. The result reveal a good performance of the artificial neural network, because its contain suitable adapting feature in the field of power system protection. The ANN depends on the range of input data used in training which must cover each working case of the power system (Normal working case and fault case) taking in to consideration the effectiveness of the system conditions upon the transmission lines. 


\section{Reference}

[1] Taher Bouthiba, "Fault Location In EHV Transmission Line Using Artificial Neural Networks", University of Science and Technology of Oran Faculty of Electrical Engineering, Int. J. APPL-Math. Compute. Sci., Vol .14, No.1, PP.69-78, 2004.

[2] A.H.Osman, Tamer Abdelazim and O.P.Malik, "Adaptive Distance Relaying Technique Using On-Line Trained Neural Network", Dept. of Electrical and Computer Engineering, University of Calgary, PP.1848-1853, 2003.

[3] P. K. Dash, A. K. Pradhan and G. Panda " A Novel Fuzzy Neural Network based Distance Relaying Scheme", IEEE, VOL. 15, NO. 3, July 2000.

[4] Gerhard Ziegler, "Numerical Distance Protection ", Principles and Application, Handbook, Siemens AG, Berlin and Munich, 1999.

[5] ALSTOM, "Protection and Automation Net Work", Guide Alstom T\&D Energy Automation and Information, Handbook, Peter Rush, Levauios-Perret France, 2002.

[6] S.A. Khaparde, S.H. Agarwal, "Adaptive Distance Relaying Using Artificial Neural Network", IEEE, PP.447-452,1993.

[7] K.K. Li, L.L. Lai and A.K. David,“ Stand Alone Intelligent Digital Distance Relay”, IEEE Trans. ON Power Systems, Vol.15, No.1, February 2000.

[8] S.H. Horowitz, A.G. phadke, J.S. Thorp, “ Adaptive Transmission System Relaying," IEEE Transsaction on power Delivery, Vol. 3, No. 4, p. 1436-1445, Oct. 1988.

[9] M.M. Saha, E. Rosolowski, J. Izykowski, "Artificial Intelligent Application to Power System Protection", Department of Electrical engineering University of Technology, POLAND, 1999.

[10] Gaganpeet ChawLa, Mohinder S.Sachder and G. Ramakrishna, "Artificial Neural Network Applications For Power System Protection", Power System Research Group, University Of Saskatchewan, IEE, PP.1954-1957 ,May 2005.

[11] A.L.O Fernandez, N.K.I Ghonaim, " A novel Approach using a FIRANN for fault detection and direction estimation for high voltage transmission lines ," IEEE Vol. 17, No. 4,pp 894-900, Oct 2002.

[12] M F Othman, M Mahfof, and D A Linkens, "Transmission Lines fault detection classifications and location using An Intelligent Power System Stabilizer ", IEEE, Honk Kong, (DRTP2004),April ,2004

[13] Richard C. Dorf , "The Electrical Engineering Handbook", Handbook, CRC Press LLC, Ch. 25, 2000.

[14] He Iiali, Duan Yugian, Li Yongli, Wang Gang and Luo Shanshan, "Distance Relay Protection Based On Artificial Neural Network" ,Tianin University, pp.515-520, November 1997.

[15] Yi Hu, Damir Novosel, Murari Mohan Saha and Volker Leitl Off, "An Adaptive Scheme For Parallel-Line Distance protection", IEEE Transaction On Power Delivery, Vol.17, No.1, PP105-110, January 2002. 